\title{
I Ciclo de Conferencias sobre Urbanismo, celebradas en el Instituto de Estudios de Administración Local
}

\section{por \\ PAULINO MARTIN HERNANDEZ}

El 10 de febrero de 1966 se clausuró en el Instituto de Estudios de Administración Local un Curso general de Información sobre Urbanismo que se desarrolló en dos partes: las clases y trabajos prácticos habituales en los Cursillos de Perfeccionamiento y un Ciclo de Conferencias.

Asistir a lo largo de todo un mes-10 de enero a 10 de febrero-a veintidós Conferencias sobre un mismo tema genérico, el Urbanismo, puede parecer, en principio, más una tarea agotadora cargada de monotonía, en especial a partir de los días iniciales, que un atrayente cometido, repleto de interés.

Sin embargo, cuantos hemos asistido a este Ciclo, podemos afirmar que no ha decaído la atención con la que se ha seguido. Sin duda, uno de los elementos que han contribuído al éxito, ha sido la extraordinaria valía de los conferenciantes: Catedráticos de Universidad, algunos; Profesores del Instituto; otros, y varios altos funcionarios especializados en estas cuestiones. Además, hay que poner de relieve la adecuada distribución del programa, en el que se encontraban temas generales junto a aspectos urbanísticos concretos; el desarrollo de muchas Conferencias, en especial las de contenido más técnico, que fueron acompañadas de abundante documentación gráfica y proyecciones de diapositivas que, en todo momento, captaron la atención de los asistentes, y los mismos coloquios con que, cada noche, terminaban las exposiciones. Por último, es necesario citar la propia formación de la mayoría de los asistentes, quienes por su profesión, tienen que vivir cada hora de los múltiples y diversos problemas urbanísticos insertos en la competencia de los $\mathrm{Mu}$ nicipios a los que sirven.

$\mathrm{La}$ asistencia de ilustres personalidades, entre las que ahora recordamos al excelentísimo señor Alcalde de Madrid, don Carlos Arias Navarro; al ilustrísimo señor Director general de Urbanismo, don Pedro Bidagor; al ilustrísimo señor Director general de Bellas Artes, don 
Gratiniano Nieto; al ilustrísimo señor Alcalde de Oviedo, a varios Subdirectores generales, etc., y el extraordinario eco alcanzado en todos los medios informativos, son buena prueba de este éxito, el cual debe impulsar al Instituto para proseguir el camino iniciado.

En el acto de clausura se leyó una carta del ilustrísimo señor $\mathrm{Di}$ rector general de Administración Local, quien no pudo asistir a las Conferencias requerido por los trabajos encaminados a ultimar el anteproyecto de Ley de Reforma de las Haciendas locales, en la que, después de mostrar el vivo interés con que había seguido el Ciclo de Conferencias, hizo presente su complacencia y felicitación a todos los que habían colaborado en el mismo.

El hecho de que el texto integro de las diversas intervenciones vaya a ser recogido en el volumen que ya se encuentra en preparación, me releva de hacer demasiado extensa esta Crónica, sin perjuicio de que intente recoger, siquiera sea con brevedad, el resumen de cada Conferencia.

La apertura de este Ciclo correspondió al Catedrático de Geografía de la Universidad de Madrid, don Manuel de Terán Alvarez, quien disertó sobre «La ciudad como forma de ocupación del suelo y de organización del espacio». Esta Conferencia, en nuestra opinión, además de su brillante exposición y del atrayente interés de su contenido, contribuyó a delimitar el campo al que estaban dedicadas las siguientes sesiones. La ciudad es la principal diana del Urbanismo actual, pero, por otra parte, existe dificultad para encontrar un concepto totalizador de la misma. «El suelo ocupado por la ciudad es sometido a una radical mutación y convertido en una nueva y original forma de paisaje». Esta ocupación está caracterizada por el alto grado de densidad de la población, la intensidad del aprovechamiento y la diferenciación interna del espacio por motivos sociales y económicos. Por otro lado, la ciudad es un eficaz instrumento para la organización del espacio que, al producir un equilibrio de fuerzas, debe tenerse en cuenta en la planificación regional. Los rapidísimos avances de la técnica, cuya velocidad se ha multiplicado diez veces en lo que va de siglo, impiden prever los resultados e incluso desfasan las realidades del planeamiento más previsor.

El Doctor Ingeniero de Montes don Manuel Martín Lobo trató del tema «El desarrollo regional en el mundo y en España», tema, por otra parte, que constituye la preocupación del conferenciante y al que ya ha dedicado otros trabajos y publicaciones. El desarrollo regional debe ser entendido en un sentido amplio que va desde un programa local hasta un conjunto regional en el ámbito internacional. En este campo es de destacar la experiencia francesa que se mueve en líneas de continua superación. Por lo que hace a España, los primeros pasos se dan con las Confederaciones hidrográficas y, más tarde, con los Planes de Badajoz y Jaén y otros Planes a nivel provincial. Actualmente, el desarrollo regional está calificado como «objetivo y directriz de nuestro Plan de Desarrollo». La última parte de esta interesante Conferencia, fue dedicada a dos técnicas que deben tenerse en cuenta al formular el 
próximo Plan de Desarrollo: «los ejes de desarrollo» que completarán los primeros logros de los polos y el «desarrollo regional rural» que «pueda contribuir a resolver los problemas de nuestra agricultura mediante un planteamiento integral e integrado».

Sobre «Crecimiento urbano y desarrollo comunitario» habló el Catedrático don José-Mariano López-Cepero, quien consideró este crecimiento como una fase avanzada de civilización técnica y expuso los problemas que el mismo comporta con el trasvase de poblaciones rurales a las urbes y los consiguientes cambios de valores y de conductas. Esta problemática exige un plan de desarrollo comunitario cuyo objetivo sea lograr el Humanes optimum. Insistiendo en el mismo tema, si bien desde otro ángulo, el Profesor don Francisco Quirós Linares expuso su Conferencia titulada «Aspectos de la población urbana», en la que destacó la diversa composición de la población atendiendo al grado de evolución de la ciudad y analizó cada uno de los elementos que intervienen en su estructura.

La Conferencia del quinto día, titulada «Naturaleza jurídica de los Planes urbanísticos», estuvo a cargo del Catedrático de Derecho administrativo don Eduardo García de Enterría, quien hizo un largo estudio del Plan, idea que-según él-pertenece a la mitología social de nuestro tiempo. El Plan por excelencia, es el urbanístico y está caracterizado por la cada vez mayor extensión del ámbito territorial, hasta el punto de que, si hoy estamos ya en presencia de Planes nacionales, no pasará mucho tiempo para que aparezcan los Planes supranacionales, e incluso continentales. El Plan se diferencia del simple Programa, en que para conseguir sus objetivos arbitra medios e instrumentales. Por otra parte, el Plan ha rectificado el contenido tradicional de la propiedad; en principio señala el paso de la propiedad rústica a la urbana, pero además regula todo el aprovechamiento del suelo urbano, y esto, de manera desigual. Se produce, por tanto, una vinculación de la propiedad a un destino, lo cual no es una limitación strictu sensu, sino un verdadero límite. Por otro lado, se produce también una vinculación de la Administración a los Planes aprobados. Por último, se plantea el problema del supuesto carácter normativo de los Planes, cuestión que parece romper los moldes tradicionales.

Don Carlos Vizcaíno Coloma desarrolló el tema «Problemas específicos del Planeamiento municipal. Racionalización del procedimiento de ejecución», en el que puso de relieve interesantes aspectos de estas cuestiones y, en especial, su reciente y aprovechable experiencia para conseguir la simplificación de procedimientos en diversas Corporaciones locales, manifestándose decidido partidario de la revisión de procesos para evitar los defectos de la rutina administrativa.

«Defensa del Patrimonio histórico-artístico» fue el título de la Conferencia a cargo del Comisario general, don Gabriel Alomar. En este Patrimonio cabe encuadrar, desde parajes de belleza singular y conjuntos urbanos, hasta los monumentos propiamente dićchos y los bienes muebles de valor artístico. La defensa de estos valores constituye, sin duda, uno de los mayores imperativos de la hora presente, porque abo- 
cados, en el futuro, a una aterradora monotonía, tan sólo se librará lo que ahora se consiga salvar de este legado del pasado.

Las cuestiones sanitarias, sin duda, ligadas de manera directa al Urbanismo, fueron estudiadas por don José-Manuel Romay Beccaría y don Enrique Mata Gorostizaga, cuyas Conferencias se titularon, respectivamente, «Política sanitaria y Planeamiento urbanístico» y «Problemas de saneamiento». Ambos conferenciantes insistieron en la necesidad de plantearse esta problemática para conseguir dos objetivos fundamentales: salud pública y bienestar social. El primero indicó la conveniencia de fortalecer la presencia de la Administración sanitaria en los Planes de Urbanismo, creando servicios de Ingeniería sanitaria, y el segundo señaló la necesidad de incrementar las inversiones con esta finalidad. Los dos pusieron de relieve el acierto que ha constituído la creación de la Comisión Central de Saneamiento.

Nuestras ciudades, construídas, en gran parte, antes de que hiciesen su aparición los vehículos de motor, no están preparadas para la corriente circulatoria cada día más intensa y que amenaza con asfixiar la vida de la ciudad. «El Urbanismo y el tráfico» fue el tema elegido por don José Luis Torroba Llorente, quien se extendió en el examen del problema y de sus posibles soluciones. Resaltó el hecho de que, en general, la solución se persigue no por el procedimiento de alterar físicamente la estructura de las redes viales, sino por el más sencillo de adoptar niveles más bajos de salubridad y amenidad.

Al Plan general dedicó su Conferencia don Francisco Javier de Mesones Cabello, bajo el título «Un ejemplo característico de Planeamiento general: la metodología de la información, análisis y prognosis; el proyecto». El futuro es la cuarta dimensión que aparece en la ciudad, por eso la labor del urbanista, comparable a la del educador, debe ser la de descubrir su vocación. Dentro de las actuales escuelas de Planeamiento, el conferenciante se inclinó por los desarrollos orgánicos monodireccionales y explicó las diversas fases de formación del Plan, completando su disertación con la proyección del Plan de Ordenación de la ciudad de Oviedo.

Aspectos concretos del Planeamiento fueron estudiados en otras dos Conferencias. Don Fernando de Terán Troyano habló sobre «Un ejemplo característico de Planeamiento parcial», señalando que la base de este Planeamiento se encuentra en la compaginación de unas óptimas condiciones de habitabilidad con una adecuada estructuración de la vida social y con una eficaz organización funcional. Para lograrlo se estructuran unidades urbanas de tamaño adecuado, libres de tráfico interior y dotadas de los correspondientes servicios comunes. Por último, se refirió a la aplicación práctica de estas ideas en el Plan del polígono «Lobete», de Logroño. Don Esteban Costa Fernández expuso el tema titulado «Planeamiento de polígonos industriales», como posibilidad de realizar en forma adecuada el desarrollo industrial, sin interferir el de los sectores residenciales. Estos polígonos exigen una dimensión determinada, dotación previa de servicios, tamaño mínimo de las parcelas, 
realización por etapas, establecimiento de centros administrativos para los servicios comunitarios, etc.

Otras dos Conferencias, singularmente interesantes, por cuanto enfocaban problemas jurídicos y de política del Suelo, fueron las desarrolladas en los días siguientes. Don José M.a Boquera Oliver habló sobre «El Patrimonio municipal de Suelo», solución prevista en nuestra Ley del Suelo, y, desde luego, más modesta que la de socializar o municipalizar el suelo urbano, fórmula propuesta en casi todos los países, pero que no ha tenido efectividad en ninguno de los del Occidente europeo. En España la creación de Patrimonios no ha seguido un ritmo acelerado, sino que, por el contrario, apenas se ha iniciado, posiblemente por la falta de recursos económicos de nuestros Municipios. No obstante, su constitución debe ser una realidad. También es interesante la configuración jurídica de estos Patrimonios, a los que el conferenciante calificó como bienes de dominio público.

«Edificación forzosa» fue el título de la charla a cargo de don Narciso de Fuentes Sanchiz. Los solares que resulten del Planeamiento deben ser obligatoriamente edificados; esta es la mejor manera de luchar contra la especulación. El reciente Reglamento de 5 de marzo de 1964 debe producir resultados en la dirección apuntada.

También se dedicaron dos sesiones a los temas de valoración del Suelo. Sobre «Técnica de la valoración del Suelo» disertó don Manuel Thomas Arrizabalaga, quien después de tratar de los cuatro valores que la Ley del Suelo establece, centró su atención en el mecanismo de la Ley para obtener las tasaciones urbanística y expectante. Los «Problemas de la técnica de la valoración agrícola en relación con la Ley del Suelo» ocuparon la disertación del Ingeniero Agrónomo don Joaquín Fernández Crespo, quien se preocupó del concepto de valor rústico en función del rendimiento de la fertilidad de los predios, aunque, hizo observar, que estos valores sufren deformaciones en las inmediaciones de las ciudades.

Una documentadísima Conferencia, titulada «El Suelo urbano: aspectos económicos y fiscales», nos of reció don Juan Antonio Lara Pol, para quien la lucha contra la especulación del Suelo debe centrarse en su raíz, impidiendo las condiciones favorables al especulador y procurando la distribución equitativa de los aumentos del valor del Suelo. En el aspecto fiscal, aludió a la legislación comparada, indicando que se debe procurar gravar tanto los rendimientos ordinarios como las plus valías.

El Secretario general del. Ayuntamiento de Madrid, don Juan José Fernández-Villa y Dorbe, habló sobre «El Derecho urbanístico en relación con el Municipio», refiriéndose, en primer lugar, a lo que denominó «Patrimonio espiritual» de las Corporaciones, para dedicarse después a analizar la totalidad de la legislación urbanística, hasta sus últimas manifestaciones con la creación de Gerencias de Urbanismo, y haciendo referencia a las aportaciones que en estas materias han realizado, tanto el Instituto de Estudios de Administración Local como los Cuerpos Nacionales. Finalmente, hizo alusión a las ciudades del fu- 
turo y a los nuevos métodos de la «Ekística» como ciencia de las agrupaciones humanas.

Las áreas metropolitanas, término que muy recientemente se ha empleado por vez primera en España, fueron objeto de diversas intervenciones. Don Emilio Larrodera López habló sobre «El Planeamiento en las áreas metropolitanas», comenzando por examinar su concepto y el proceso de formación, para entrar después en el análisis de su planeamiento, en el que aludió, especialmente, al centro de la constelación de agrupaciones, a los sistemas de comunicación de éstas y a las zonas exteriores. Terminó refiriéndose a la posible creación de nuevas ciudades para resolver los problemas que las actuales plantean.

Podría decirse que la Conferencia del día siguiente, a cargo de don Julio Cano Lasso, titulada «El polígono de descongestión de Toledo como primera actuación del desarrollo urbanístico del Valle del Tajo», fue una continuación de la anterior, por cuanto la creación de este polígono obedece a la necesidad de descongestionar Madrid, lo cual se pretende hacer mediante un Plan a escala regional. Las condiciones territoriales son favorables para un desarrollo de esta naturaleza, constituyendo esta primera actuación un núcleo autosuficiente para 90.000 habitantes y 25.000 puestos de trabajo industrial, concebido como primer elemento de un sistema lineal de núcleos que pueden reunir más de un millón de habitantes.

Tocó cerrar este Ciclo de Conferencias al Maestro don Luis Jordana de Pozas con la titulada «Las áreas metropolitanas en España y en el extranjero», la cual constituyó una brillante exposición, tanto por las interesantes referencias a las novísimas realidades de distintos países, como por el examen de la doctrina, la realidad y el Derecho positivo en relación a España. Terminó aludiendo al problema de las estructuras territoriales cuya actualización es una apremiante necesidad pública.

A continuación, el Director del Instituto, don Carlos Ruiz del Castillo, pronunció un discurso. En él hizo referencia a la labor ya realizada por el Instituto en relación con el Urbanismo, y puso de relieve cómo, con el Curso que se clausuraba, «ha quedado abierto un camino fecundo -el del diálogo en la propia interdependencia de los temas que afectan al Urbanismo-y que invita a la marcha común de un equipo fraterno que enlaza a la Administración con la Sociedad, el Municipio y el Estado, a los técnicos especialistas y a los hombres procedentes del campo social, imbuídos de saberes humanísticos». Agradeció las múltiples colaboraciones y terminó brindando el esfuerzo del Instituto «a los logros de un Urbanismo a la escala del hombre y a la altura de nuestro tiempo».

Los actos finales de este primer Ciclo de Conferencias, que coincidieron con la terminación del Curso General de Información de Urbanismo. fueron la entrega de Certificados y una cordial reunión de despedida en la que estuvieron presentes todos los participantes. 